\title{
Relationship Between Demand for Emergency Room Care in Newborns and Maternal Counseling About the Main Events in the Neonatal Period
}

\author{
Angelo Carneiro Bonadio, ${ }^{1,}$, Raquel Aparecida Oliveira ${ }^{2}$ \\ ${ }^{1}$ Pediatric Department, Pontificia Universidade Catolica de Sao Paulo, Sao Paulo, Brazil \\ ${ }^{2}$ Nursing Department, Pontificia Universidade Catolica de Sao Paulo, Sao Paulo, Brazil \\ Email address: \\ angelo_bonadio@hotmail.com (A. C. Bonadio), raoliveira $a$ pucsp.br (R. A. Oliveira) \\ ${ }^{*}$ Corresponding author
}

\section{To cite this article:}

Angelo Carneiro Bonadio, Raquel Aparecida Oliveira. Relationship Between Demand for Emergency Room Care in Newborns and Maternal Counseling About the Main Events in the Neonatal Period. American Journal of Pediatrics. Vol. 5, No. 4, 2019, pp. $234-239$.

doi: 10.11648/j.ajp.20190504.22

Received: August 29, 2019; Accepted: September 25, 2019; Published: October 31, 2019

\begin{abstract}
The neonatal period, between the date of birth and twenty-eighth days after birth, is a time when parents face many doubts and anxiety. Even previous experiences are not enough to avoid or diminish parental anxiety. Therefore, parents commonly take their newborns to emergency units to care for simple health disorders. Despite reassuring the parents, this custom can be harmful to the newborns because they get exposed to a wide pathogen circulation environment. Since immunity from vaccination is still not complete, as well as due to the age-specific immunodeficiency, this behavior is risky to the newborns. The study aims to reduce the newborns' care flow to emergency units using educational interventions in maternity wards. Methodology: This is a descriptive, quantitative and qualitative study, that evaluate the impacts of an educational intervention done by pediatricians, in the municipal maternity of Salto de Pirapora, in the Brazilian countryside. It is based in individual questionnaires, applied before the educational intervention to 99 mothers and newborns' accompanying parties, in order to understand the most frequent concerns about the main health problems during the neonatal period. A dialogical educative intervention was done in the maternity regarding how and when the newborn should be taken to an emergency unit. The same mothers answered an intervention evaluation questionnaire after the neonatal period; the data of newborns' care flow towards emergency units before and after the intervention was also compared. The results show that there was a reduction on the emergency services demand. The proportion of patients going to the emergency room was $36.7 \%$ on the last year and $30.56 \%$ two years before the intervention. The reduction reached about $12.33 \%$ after the intervention, an average reduction of $21.3 \%$. The biggest concerns of the participants were: fever $65.63 \%$, choking or suffocating $39.58 \%$ and constant crying $33.33 \%$.
\end{abstract}

Keywords: Emergency Care, Neonatology, Pediatrics, Health Education

\section{Introduction}

The lack of information about the main complications in the neonatal period can generate anxiety in family members, and this circumstance becomes an important catalyst for seeking care in the emergency rooms. [1,2] The act of taking a newborn to the emergency service can have some negative consequences. In these environments there is usually wide circulation of aggressive pathogens. [3] Neonates' immunosuppression, coupled with incomplete vaccination coverage, leave them exposed to contagion with such microorganisms circulating in this environment. [4]

Financial costs should also enter into the equation. There is usually an economically active person that must be available to accompany the minor in the hospital, leading to losses in the economic activity. [5] Each patient seen at the hospital generates costs regardless of the complexity of the case [1] and those poorly spent resources end up interfering with alternative investments to improve service quality.

A study in Spain found that $91.3 \%$ of families opt to take 
the newborn directly to the emergency room. [6] A study conducted in Brazil indicates that only $18.9 \%$ of parents prefer to look for first care in the primary health care (PHC) network. It was found that $63.5 \%$ of the children taken to the emergency services had health problems that could be solved in this level of care. [7] Similar results were observed in Singapore, where $60 \%$ of cases seen in the emergency room were not characterized as urgent. [8] Additionally, a study done in Chile shows even more striking data, as $90 \%$ of the patients surveyed had conditions that could have been satisfactorily resolved in low-complexity care. [9]

A study conducted in Canada at a pediatric emergency unit points out that the most common complaints were jaundice, difficulties in breathing, eating problems and irritability, while the most frequent diagnoses were: normal status $(27 \%)$, jaundice $(25.3 \%)$, eating problems $(7.8 \%)$, and infection (7.2\%). [10] These data were reinforced by a study conducted in Portugal where the main complaints were jaundice (14.5\%), excessive crying (13.6\%), rash $(7.3 \%)$, nasal congestion $(6.7 \%)$ and cough $(5.9 \%)$. The main diagnoses found in the same study were: normal status (26.1\%), colic (11.3\%), physiological jaundice (8.2\%), and upper airway infection (7.3\%). [2] A study carried out in Turkey shows that the main complaints were jaundice $(23.4 \%)$, irritability $(9.5 \%)$ and vomiting $(7.1 \%)$, while the most common diagnoses were: normal newborn $(33.9 \%)$, jaundice (13.2\%) and colic (5.8\%). [11]

Several factors, such as first pregnancy, low maternal age, early postnatal discharge, low family income, single mother, low parental educational levels and mainly the caregivers' insufficient knowledge about newborn care are affecting an adequate newborns' use of pediatric emergency departments. $[1,6,8,10]$

Maternal counseling could therefore decrease spontaneous demand for emergency care, by exposing major complications and explaining under what circumstances the newborn should be brought to the emergency room. $[1,11]$ These initiatives give mothers the opportunity to express their own questions, therefore facilitating understanding and improving their interest. [12]

\section{Methods}

The study was done regarding health education effects, through a controlled non-randomized and not blinded trial. It used quantitative, qualitative, documentary research and interventional approaches. The interventions on the study group were conducted in November and December 2018 and January and February 2019, while the control group encompassed the same months in the two years prior to the intervention.

The study was developed at the Municipal Maternity Hospital and Santa Casa de Salto de Pirapora Hospital. Salto de Pirapora is a Brazilian municipality with 44,972 inhabitants in the countryside of the state of São Paulo. These facilities are the only maternity and emergency care units available to residents in the county. The 2010 Municipal
Human Development Index for the municipality was 0.729 . [13]

The first stage involved 99 mothers who had their deliveries at the Salto de Pirapora Municipal Maternity. The mothers were individually interviewed while they were still hospitalized, following a semi-structured script about their main concerns regarding changes in their children's health and how they would approach such situation.

This step of the process also included an educational intervention using dialogicity, [12] after the interview. The intervention was based on the concerns and prior knowledge of mothers that were raised in the interview. The participatory approach acknowledged the participants' lore and doubts, involving them in the discussion. After the discussion the group elicited a collective opinion able to be incorporated into daily practice. If the concept agreed upon by the group was wrong, the researcher had the role of acting as moderator, guiding them towards the correct path. This methodology enables interpersonal interactions as well as allows paying attention to the information exposed, facilitating the understanding and retention of contents. [14] At the end of the activity, the researchers distributed an educational leaflet referring to the main topics covered in the activity.

The second stage included an interview after the end of the 28 days of the neonatal period in order to assess the improvement gained by the mothers. They were asked if they had enjoyed the experience and if they believed they were now more prepared to deal with the main complications that may arise. In addition, there were questions regarding any complications during the neonatal period and how they were approached.

In the third stage, a documentary research was carried out through the Santa Casa de Salto de Pirapora Hospital's emergency room medical records' system, collecting information regarding newborns care flow in two moments:

1. Records corresponding to two years prior to the intervention regarding the number of attendances of newborns in the emergency room;

2. Records corresponding to the post-intervention period referring to the number of attendances of newborns in the emergency room until each of the research participants reached 28 days of life.

The results of the interviews after the neonatal period were statistically compared with the emergency care flow after intervention to analyze the project impacts on the public health system and on the mothers' personal perception.

\section{Results}

The intervention at the maternity hospital began in early November 2017 and lasted until the last day of February 2018. During these 4 months, data were collected in individual interviews alongside the educational intervention. At this stage the research collected information regarding marital status, education, obstetric antecedents, as well as questions about the main diseases that newborns could face. 
One mother refused to participate in the survey, promptly respecting her decision.

The initial intention was to perform the educational process in an specific room, separated from newborns, to allow mothers to have full attention to the information transmitted in the activity. However, this proposal proved to be unfeasible, because some mothers had pain and displacement problems. Additionally, the time spent in the activity by a few groups proved to be extensive, generating great anxiety and lack of attention in the population under study, even after knowing that the nursing staff or their companions were assisting their children.

As a result, the application of the activity was reformulated. The educational intervention began to happen in the rooms, where there were up to four maternal beds and space for their companions. In this way, new listeners had the opportunity to learn and replicate the knowledge learned. Activity was therefore no longer limited to mothers, although they were the center of attention.

The individual responses obtained from the mothers were categorized by themes and grouped by similarity. Ninety-nine mothers were interviewed prior to the educational activity. A large percentage of them, $72.72 \%$, belonged to the age range between 20 and 34 years. The maternal proportion of elderly pregnant women was $13.13 \%$, while adolescents were a $14.14 \%$.

The educational level of the population was concentrated among those with incomplete $(23.23 \%)$ and complete high school (39.39\%). A small percentage (7.07\%) had incomplete higher education and another $7.07 \%$ had completed higher education. The percentage of individuals with up to eight schooling years was $23.23 \%$. The largest share of the population was between eight and eleven years of study, $62.62 \%$. Single mothers were $13.13 \%$ of the sample. Participants in stable union corresponded to $86.87 \%$. Mothers having their first child were $39.39 \%$, while the remaining $60.61 \%$ already had one child or more.

The intervention in smaller groups presented less opportunity for interaction and exchange of experiences, while lack of attention was more frequent in larger ones. The distribution of active time was related to the behavior of the groups. In the most active groups where the participants interacted more with each other and presented more previous knowledge and doubts, discussions lasted longer. The maximum time allocated to the same group was 120 minutes (3.70\% of the total number of groups). The mode of time spent to perform the intervention was 60 minutes $(29.63 \%$ of cases). Groups in which there was little opportunity to exchange experiences and interactions were accordingly shorter in time. In the shortest groups $(24.07 \%)$ the activity lasted 20 minutes. A duration of 30 minutes happened in $27.78 \%$ of groups while it lasted 40 minutes in $14.81 \%$ of groups. The average time each person participated in the activity was 41 minutes and 20 seconds.

Each participant posed an average of 3.38 questions. Mothers with higher education were well attentive and participative in the activity, even though the higher level of education did not reflect the degree of previous knowledge. The group of higher education had questions slightly above the overall average, 3.43. The degree of complexity in some questions of these mothers was remarkable. Mothers with incomplete elementary school had the lowest number of questions, an average of 2.85 questions per participant. Mothers who had experience due to previous pregnancies expressed 3.32 doubts per participant, a higher average than those who had their first child, which had 3.21 questions per mother.

At least a quarter of participants expressed doubts on the following subjects: fever, choking or suffocation and constant crying. The large number of mothers who expressed doubts about how to classify and how to act in case of fever is noteworthy. Even the group of more experienced mothers with children, expressed greater concern about this topic (66.67\%) compared to primiparous mothers (64.1\%). None of the 99 mothers, regardless of their educational level, was able to correctly establish which temperature merited medication.

The complete distribution of questions is presented in table 1.

Table 1. Frequency of questions, Salto de Pirapora, November 2018 to February 2019.

\begin{tabular}{|c|c|c|c|c|}
\hline Pathology & Percentage & Total of questions & Questions in primipara & Questions in mothers with other children \\
\hline Fever & 65,66 & 65 & 25 & 40 \\
\hline Choking/suffocation & 39,39 & 39 & 17 & 22 \\
\hline Constant crying & 33,32 & 32 & 16 & 16 \\
\hline Allergies & 24,24 & 24 & 10 & 14 \\
\hline Rhinorrhea/Cough & 24,24 & 24 & 7 & 17 \\
\hline Breastfeeding disorders & 19,19 & 19 & 6 & 13 \\
\hline Reflux/Vomit & 14,14 & 14 & 2 & 12 \\
\hline Dyspnea & 13,13 & 13 & 4 & 9 \\
\hline Fall & 9,09 & 9 & 1 & 8 \\
\hline Pain & 7,07 & 7 & 6 & 1 \\
\hline Jaundice & 7,07 & 7 & 2 & 5 \\
\hline Convulsion & 5,05 & 5 & 4 & 1 \\
\hline Disorders in elimination & 5,05 & 5 & 0 & 5 \\
\hline Mononucleosis & 4,04 & 4 & 2 & 2 \\
\hline Cardiopulmonary disease & 3,03 & 3 & 0 & 3 \\
\hline
\end{tabular}




\begin{tabular}{lllll}
\hline Pathology & Percentage & Total of questions & Questions in primipara & Questions in mothers with other children \\
\hline Insect bite & 3,03 & 3 & 1 & 2 \\
Other & 30,30 & 30 & 13 & 17 \\
TOTAL & 315 & 122 & 193 \\
\hline
\end{tabular}

A new interview was conducted after 28 days of birth. In this second stage 93 mothers answered the questions by telephone, while 6 of the participants could not be contacted. The answers collected from the questionnaires were grouped into themes and quantified in frequency. The topics addressed referred to individual perceptions regarding the way the intervention collaborated with newborn care. The research used Content Analysis [15] to analyze the records.

When asked about their feelings regarding the care of their children, most of the interviewed mothers expressed their optimism in a variety of ways, corresponding to $69.64 \%$ of the total. All participants expressed their approval when asked about the activity's validity and whether it had added anything to their daily practice. Some mothers also reported that they were now multipliers of the knowledge acquired in the maternity ward. The participation of accompanying parties as listeners was positive, as some mothers reported that they had difficulty when dealing with their sick children, but their company not only knew how to handle the situation, but also was instrumental in disseminating what they had learned.

There was a question regarding if the newborns had any health problems after leaving the hospital environment. There were 72 complications in 61 patients, the largest number of them were choking-related cases. This problem was cited by $18.06 \%$ of the total number of mothers. All others are shown in table 2.

Table 2. Events in the neonatal period, Salto de Pirapora, from November 2018 to February 2019.

\begin{tabular}{lll}
\hline Events, neonatal period & Number & Percentage \\
\hline Choking & 13 & 18,06 \\
Colic & 11 & 15,28 \\
Nasal Obstruction & 11 & 15,28 \\
Reflux & 10 & 13,89 \\
Jaundice & 6 & 8,33 \\
Cough & 6 & 8,33 \\
Skin spots & 3 & 4,17 \\
Skin irritation & 2 & 2,78 \\
Frequent crying & 2 & 2,78 \\
Fever & 2 & 2,78 \\
Umbilical hernia & 1 & 1,39 \\
Sneezing & 1 & 1,39 \\
Nausea & 1 & 1,39 \\
Diminished defecation & 1 & 1,39 \\
Tachypnea & 1 & 1,39 \\
Persistent umbilical cord & 1 & 1,39 \\
TOTAL & 72 & 100 \\
\hline
\end{tabular}

Mothers were invited to express their feelings about the complications they needed to face. Even with the guidance given in the activity, most mothers (57.78\%) did not feel fully able to deal with the situation. Next, it was asked how mothers dealt with complications. The initial stance followed the guidance provided, showing conducts in order to postpone the search for care in a health unit. An attentive observation of the evolution of the case was made by almost half of the population studied. Of the 72 reported complications, the mothers at home handled 62, without needing to go to the emergency unit, representing $86.11 \%$ of the cases.

However, in ten cases, the parents decided afterwards to go to health facilities in the municipality. The most frequent diagnosis was mild respiratory problems, present in five cases. In this situation the recommendation was only inhalation and nasal cleansing. Other cases were jaundice, natural peeling of the skin, colic of the newborn and dermatitis. Only the case of jaundice needed hospitalization for treatment. One of the mothers reported that despite taking her child to the emergency room, the content learned was important to make her less concerned.

In order to have a more solid analysis, the research compared information from the two years prior to the intervention. November data was discarded due to lack of reliable information in the hospital system. The data show that two years before, 72 individuals were born, and also in the year before the study there were 109 babies born, while the number of births in the year of the study was 71. As the study was aimed to analyze the first 28 days of life, the number of patients in the emergency room was monitored until the twenty-eighth of March. The total demand for neonatal care in the emergency room from December 2016 to February 2017 totaled 22 patients; from December 2017 to February 2018, 40 patients; and during the period of this study there were 9 newborns showing up. The compiled data are presented in table 3 .

Table 3. Demand for emergency room.

\begin{tabular}{llll}
\hline December, January and February & $\mathbf{2 0 1 6 - 2 0 1 7}$ & $\mathbf{2 0 1 7 - 2 0 1 8}$ & $\mathbf{2 0 1 8 - 2 0 1 9}$ \\
\hline Demanded emergency services & 22 & 40 & 9 \\
Not demanded emergency services & 50 & 69 & 62 \\
TOTAL & 72 & 109 & 71 \\
\hline
\end{tabular}

Based on the data presented in table 3, when comparing the intervention period with data from the previous year, the analysis indicated that the intervention was significantly relevant to decrease the attendance in the emergency unit, showing a value of 0.001 in the Fisher exact test. This also applies to the comparison of the data from two years prior to the study, resulting in a statistical significant Fisher's exact test value of 0.014 . The proportion of those who sought emergency care was $36.7 \%$ in the year prior to the intervention and $30.56 \%$ two years earlier and it was reduced to $12.68 \%$ after the intervention, implying an average reduction of $21.3 \%$ or 15.12 patients in the year of study.

\section{Discussion}

The average time of participation for each person in the 
activity was approximately 40 minutes. In those cases in which the orientation was an interaction only between the mother and the researcher or collaborator, the approximate time was reduced to 30 minutes.

After analyzing the data, it can be stated that giving information related to the main complications and regarding the way they should be addressed, had a positive impact, and that intervention decreased the demand for the emergency service by $21.3 \%$. Mothers said they were more capable in handling situations, gaining a positive perception about care.

The situation faced when their children are sick still worries $58 \%$ of mothers, but they have developed a greater ability to intervene at home. In this way, they reduce the exposure of their children to environments with greater circulation of pathogens, such as hospitals. Even though the real-life experience of situations that were previously approached theoretically still generates anxiety and expectation, this fact does not disqualify the activity. The intervention succeeded in better enabling mothers to identify situations that would generate demand for the emergency service, allowing $86.11 \%$ of the complications to be cared at home. The World Bank report corroborates that rising levels of education make the individuals more prepared to promote both their own health as well as the health of their families. [16]

The decrease in demand for higher complexity care also reflects savings in the municipality costs, stopping the expense of hospital supplies for patients with less serious health conditions, thus leaving more time available for health professionals to spend in cases that require more attention. Increasing education and increasing information are factors responsible for reducing the economic burden provoked by patients, according to the World Bank. [16]

All mothers approved the intervention model. By applying this activity at the bedside, allowing mothers to stay close to their children, it decreased a factor of inattention and allowed the activity to be expanded to the companions. This approach has proven to be effective, as many people related to the newborns have learned how to act on major complications during this period. These accompanying parties became a source of support for mothers, also acting as multipliers of the acquired knowledge.

Despite working with the neonatal age group, the complications and orientations were not restricted to this age group. Thus, the benefits previously highlighted may extend to older children, expanding their impact on health indicators. This fact is suitable to be measured in future applications of the study model.

Even though it does not appear with the same relevance in the researched literature, the presence of concerns and questions regarding choking was quite present in the studied population, being mentioned by $39.39 \%$ of mothers. The main doubts referred to the correct procedure to revert choking. It was the most common complication among newborns followed throughout the study, encompassing 13 individuals. All cases were satisfactorily reversed using the given orientation, thus demonstrating the positive impact of the guidelines.

It should be noted that a large number of mothers have apprehension and uncertainty about fever, a common event in childhood. It was cited by $65.66 \%$ of mothers regardless of their previous experience with other children. Health professionals continue to be the largest source of information for family members. There is a widespread lack of knowledge regarding the normal temperature value, the proper measurements and correct use of temperaturereducing measures. [17] This fact expresses that the issue needs to be better addressed by professionals providing child health care. Other quite prevalent subjects are: choking or drowning, constant crying, allergy and runny nose or cough.

This work serves as a template for the implementation of similar projects in other institutions. The educational activity has a low cost of implementation, as it can use the health unit's own staff for its execution. The hospital must bear exclusively the costs of making educational leaflets, with the aim on reinforcing the main topics addressed, expanding the promotion of home care and the ability of family to behave actively in the health situation.

The elaboration of an educational leaflet facilitated the promotion of better understanding and refreshing of the content addressed in the educational activity. Explanatory leaflets are able to guide home care actions, in addition to contribute to the development of the caregivers' ability to intervene regarding a complication. Considerable portion of the leaflets distributed are read, and about half of them are passed on to people who are also responsible for carrying out care. [18] It is important that this vehicle of information has an adequate layout, vocabulary and content in order to be able to properly perform its role.

The literature on guidance provided by pediatricians in maternity hospitals is limited. Health education is more present in the nursing area, predominantly addressing general care, focused on hygiene and breastfeeding. This finding may suggest deficiencies in the instruction regarding changes in newborns' health as well as the lack of an appropriate approach for the responsible adults. Those deficiencies are suitable to be reversed by both pediatricians and nurses.

In addition to addressing questions raised by mothers, the activity included pre-stipulated topics for discussion, due to their frequent presence in the literature. The establishment of these both fixed and variable items, also not limited to general care, was useful as it broadened the scope of the guidelines given by pediatricians. It promoted a change in the practice of these professionals, expanding their educational dimension.

All pediatricians involved in performing the activity were surprised by the lack of knowledge of the assisted population, while at the same time considered as positive the implementation of the guidelines. Raising the educational level of family members contributes to the development of health promotion capabilities and enables them to act in the face of complications with newborns.

Some limitations of the study should be pointed out. The lack of knowledge of complications in the years prior to the 
application of the activity impeded the analysis of the influence of the educational intervention regarding different pathologies. A longer intervention period could be useful to provide more data regarding the seasonality of complications and the influence of this activity throughout the year. The study did not measure a likely impact on decreasing opportunistic infections acquired in a hospital setting.

\section{Conclusion}

The empowerment of mothers through dialogicity proved to be efficient, allowing a good retention of the contents as well as to the adequate implementation of recommendations regarding the main complications in the neonatal period. The redesign of the activity in order to be carried out at the bedside, allowed increasing the number of people close to the newborn to be trained, thus expanding its reach. Mothers considered to have improved their knowledge through the activity, something that was reflected in the home care given in $86.11 \%$ of the complications.

Compared with the average of the previous two years, the intervention made during the present study showed efficiency in a $21.3 \%$ reduction of the demand for care in the emergency room. This decrease is linked to financial savings derived from the decreased use of hospital supplies and better utilization of health professionals.

\section{References}

[1] Kubicek K, Liu D, Beaudin C, et al. A profile of non-urgent emergency department usage in an urban pediatric hospital. Pediatr Emerg Care. 2013; 28 (10): 977-984. doi: 10.1097/PEC.0b013e31826c9aab.A.

[2] Calado CS, Pereira AG, Santos VN, Castro MJ, Maio JF. What brings newborns to the emergency department?: a 1-year study. Pediatr Emerg Care. 2009; 25 (4): 244-248. http://pesquisa.bvsalud.org/portal/resource/pt/mdl-19382329.

[3] Nogueira PSF, Moura ERF, Costa MMF, Monteiro WMS, Brondi L. Nosocomial infection profile at an university hospital. Rev Enferm UERJ. 2009; 17 (1): 96-101.

[4] Vaz FAC, Diniz EMA, Ceccon MEJR, Krebs VLJ. Neonatology. (Schvartsman BGS, Maluf PTJ, eds.). Barueri: Manole; 2011.

[5] Brazilian Institute of Geography and Statistics. Minimal social Indicators: concepts. IBGE. https://ww2.ibge.gov.br/home/estatistica/populacao/condicaod evida/indicadoresminimos/conceitos.shtm. Published 2019. Accessed June 17, 2019.

[6] Perez Solis D, Pardo de la Vega R, Fernandez Gonzalez N, Ibanez Fernandez A, Prieto Espunes S, Fanjul Fernandez JL. Neonatal visits to a pediatricemergency service. An Pediatr. 2003; 59 (1): 54-58. doi: 10.1016/S1695-4033(03)78148-X.

[7] Kovacs MH, Feliciano KVO. Access to basic care for children seen at emergency departments. J Pediat (Rio J). 2005; 81 (3): 251-258.

[8] Kua PHJ, Wu L, Ong ELT, et al. Understanding decisions leading to nonurgent visits to the paediatric emergency department: caregivers' perspectives. Singapore Med J. 2016; 57 (6): 314-319. doi: 10.11622/smedj.2016023.

[9] Mendez E. B, Herrera L. P, Guerra M. H, Dattas L. JP, Munoz H. B, Velasco B. J. Structure of the paediatric population attending the Emergency Service. Roberto del Rio Children's Hospital. Rev Chile Pediatr. 2005; 76 (3): 259-265. doi: 10.4067/S0370-41062010000100001.

[10] Millar KR, Gloor JE, Wellington N, Joubert GI. Early neonatal presentations to the pediatric emergency department. Pediatr Emerg Care. 2000; 16 (3): 145-150.

[11] Batu ED, Yeni S, Teksam O. The factors affecting neonatal presentations to the pediatric emergency department. J Emerg Med. 2018; 48 (5): 542-547. doi: 10.1016/j.jemermed.2014.12.031.

[12] Freire P. Autonomy Pedagogy: Knowledge necessary to educational practice. 25th ed. Sao Paulo: Paz e Terra; 1996.

[13] Brazilian Institute of Geography and Statistics. Salto de Pirapora. IBGE. https://cidades.ibge.gov.br/brasil/sp/salto-depirapora/panorama. Published 2019. Accessed June 17, 2019.

[14] Pelicioni MCF, Mialhe FL. Education and health promotion: Theory and practice 2a. Sao Paulo: Santos; 2016.

[15] Bardin L. Content analysis. Sao Paulo: Edition 70; 2002.

[16] World Bank. World Development Report 1993 Investing in Health. Washington; 1993.

[17] Gomide ACM, Silva RM, Capanema FD, Goncalves LAO, Rocha RL. How parents deal with the child's fever: influence of beliefs, knowledge, and information sources in the care and management of fever in children - systematic review of the literature. Rev Médica Minas Gerais. 2014; 24 (2): 180-185. doi: $10.5935 / 2238-3182.20140050$.

[18] Nascimento EA, Tarcia RML, Magalhaes LP, Soares MA de L, Suriano MLF, De Domenico EBL. Educational pamphlets on health: a reception study. Rev da Esc Enferm. 2015; 49 (3): 432-439. doi: 10.1590/S0080-623420150000300011. 